\title{
Exploring the Nuances of 'Wickedness' in Information Systems Development
}

\author{
Stephen McCarthy \\ Cork Univ. Business School \\ \& Infant Research Centre \\ Stephen.McCarthy@ucc.ie
}

\author{
Paidi O'Raghallaigh \\ Infant Research Centre \\ University College Cork \\ P.OReilly@ucc.ie
}

\author{
Ciara Fitzgerald \\ Frédéric Adam \\ Cork Univ. Business School Cork Univ. Business School \\ University College Cork University College Cork \\ CFitzgerald@ucc.ie $\quad \underline{\text { FAdam@ucc.ie }}$
}

\begin{abstract}
Information Systems Development (ISD) practice is an inherently challenging undertaking, as exemplified by the high rate of ISD project failures. The scale of the challenge is often heightened in distributed environments where ISD practitioners can face considerable complexity, uncertainty, and contention. The concept of 'wickedness' epitomizes such challenges. However, ISD literature has yet to fully explore the nuances of wickedness found in ISD practices within distributed environments. To address this gap, we use a theoretical framework to analyze case study findings from an interdisciplinary connected health project. In particular, we break open the social aspects of wickedness and explore their impact on shared understanding and shared commitment in ISD projects. The paper highlights the implications that these nuances have for group decision-making in distributed ISD project teams.
\end{abstract}

\section{Introduction}

Information Systems Development (ISD) is a challenging practice with numerous decision points [1, $2,3]$. For instance, ISD projects are often characterized by tight deadlines, constrained resources, fluid team boundaries, temporary membership, and crossfunctionality $[1,4,5]$. In light of these challenges, it is hardly surprising that the rate of ISD project failure remains stubbornly high [6]. Moreover, the challenge is often heightened when teams are geographically, temporally, and organizationally dispersed [7]. Team performance in such distributed environments rests on the ability of team members to reach a shared understanding of and a shared commitment to the ISD practice [8, 9]. However, social complexity, uncertainty, and contention can impede the attempts to collaborate and make decisions [7, 10]. The impact of these social aspects remains underexplored and this gap demands further research in order to help address ISD project failure $[8,11]$.

Social complexity, uncertainty, and contention in group decision-making epitomize the concept of 'wickedness' [12, 13, 14, 15]. Wickedness describes seemingly irreconcilable differences between social groups involved in decision-making processes, where the information needed to arrive at a solution is incomplete and always changing. In particular, wickedness is prevalent in situations involving large numbers of stakeholders with different roles, interests, and values $[12,15]$. This social plurality can result in 'fragmentation', where individuals' perspectives, understandings and intentions are dispersed rather than unified [13]. Fragmentation is assumed to have a negative effect on decision-making and ultimately on the ability of teams to reach a shared understanding and a shared commitment. However, despite the concept's potential for explaining sources of ISD project failure, wickedness has received scant attention in extant ISD literature.

In this paper, we therefore aim to investigate the following research question: what are the nuances of wickedness that occur within distributed ISD environments and how do these impact the ability of project teams to reach a shared understanding and $a$ shared commitment? In investigating this question, we present empirical evidence from the in-depth case study of an interdisciplinary connected health project. Interactions between the project team members helped deliver a quality solution which project sponsors felt exceeded initial project objectives. Nevertheless, considerable challenges were faced by the diverse team members in reaching a shared understanding of and a shared commitment to the activities required in building the solution. The Typology for Organizational ISD Practice as presented by McCarthy et al [16] is used to describe and explain project team interactions and activities. This lens lays the foundation for exploring the nuances of wickedness that emerged during group decision making within the distributed ISD environment. We extend the literature by breaking 
open the social aspects of wickedness in the realm of ISD practice. We also highlight the practical and theoretical implications of wickedness in diverse ISD project teams.

The paper is structured as follows: Section 2 provides a literature review, and Section 3 outlines the theoretical development. Section 4 describes the research design and Section 5 describes a key vignette from the case study. Section 6 discusses findings from this vignette, while Section 7 offers a conclusion.

\section{Literature Review}

The practice of ISD is an inherently social activity which often requires the involvement of numerous stakeholder groups in developing solutions that satisfy the needs of the same and other groups [10]. ISD practice is often directed by group decision-making processes involving interdisciplinary teams. Moreover, group decision-making processes are increasingly taking place in 'distributed environments' where team members are geographically, temporally, and organizationally dispersed [7]. Such distributed ISD project teams are characterized by unique attributes which can hinder their performance unless properly managed [4]. For instance, project teams are typified by fluid team boundaries, temporary membership, and cross-functionality [4]. More recent advances in ICT such as high-speed internet and videoconferencing have enabled distributed collaboration on a scale previously unimaginable in ISD. This has led to the creation of new forms of organizing in which practitioners can collaborate across distributed environments with relative ease [7, 17, 18]. However, understanding of the underlying challenges in distributed ISD environments remains nascent [7].

Previous studies [5, 8, 19, 20, 21] have found shared understanding to be pivotal to the success of ISD practices involving interdisciplinary team members. Shared understanding can be defined as where "people concur on the value of properties, the interpretation of concepts, and the mental models of cause and effect with respect to an object of understanding" [22, pg. 115]. Shared understanding enables interdisciplinary teams to effectively collaborate and share knowledge around both the ISD process and content through a participatory and dialogical approach [23]. In contrast, the absence of shared understanding can potentially lead to unintended consequences for the ISD process such as late changes to requirements, rework, delays, and wasted resources [5]. Factors which have been found to affect shared understanding include organizational structures, interests, culture, and division of resources $[21,23,24]$.
Meanwhile, shared commitment goes beyond shared understanding, and can be defined as the agreement of team members to commit time, effort, and resources in line with proposals that have gained shared understanding $[13,24]$. Studies have shown the importance of shared commitment to system success [13], and engagement in online communities [9]. The concepts of shared understanding and shared commitment are interdependent as both affect an interdisciplinary team's ability to successfully conduct a practice [13]. While shared commitment cannot arise in the absence of shared understanding, shared understanding alone is not enough. Briggs, et al. [24] points towards factors that impede shared commitment among interdisciplinary teams: differences in mental models, differences in information, differences in meaning, differences in goals, and differences in tastes.

Meanwhile, 'wickedness' as a concept is attributed to Rittel and Webber [12] and was used to describe problems where shared understanding and shared commitment are extremely difficult if not impossible to achieve. Wicked problem are complex (e.g. multifaceted, interconnected, and unique), uncertain (e.g. ill-structured, ill-defined, and indeterminable), and contested (e.g. subject to differences in interpretations) $[12,13,14,15]$. Wickedness can emerge from social, technical, and domain aspects which impede the creation of clear and agreed solutions due to fragmentation among social groups [13]. 'Tame problems' are provided as a counterexample to describe problems where the problem-solver has all the information requisite for understanding the problem, articulating the mission, and designing solutions which can be judged objectively as right or wrong. The problems addressed by ISD are often wicked in nature as they involve inherent complexity, uncertainty, and contention [25, 26]. For instance, ISD project teams must analyze complex workflows, address uncertainty around system requirements, and manage contention in relation to stakeholder interests.

Our ability to understand wickedness appears to be fundamental to effective group decision-making in distributed environments. However, to date wickedness has received limited attention in ISD literature. Given the social nature of ISD, this paper focuses primarily on the social aspects of wickedness. In particular, we focus on how the presence of pluralistic structures, identities, and cultural values in social groups contribute to wickedness [c.f. 12, 15], and how this in turn affects shared understanding and shared commitment in practice. The next section presents the paper's theoretical development for understanding wickedness in distributed environments. 


\section{Theoretical Development}

This paper further refines an evolving theoretical framework called the Typology for Organizational ISD Practice, first presented in McCarthy, et al. [16]. The typology offers a lens for describing and explaining the team interactions and activities that take place during the conduction of ISD practices within complex environments [c.f. 27]. In particular, it focuses on the interplay between the macro-level factors of structure, identity, and culture and team members' micro-level interactions in practice. It is suggested that this interplay can give rise to wickedness that impacts the ability of teams to reach a shared understanding and a shared commitment. The rationale for selecting this theoretical lens is therefore that it lays the foundation for exploring nuances of wickedness in group decisionmaking within ISD practices.

The typology draws on insights from literature including the seminal works of Parsons' General Theory of Action Systems [c.f. 28], Bourdieu's Theory of Practice [c.f. 29], and more recent Sociomateriality literature [e.g. 30, 31, 32] as a sensitizing device for research on how humans and objects come together in practice. The typology assists the researcher in describing and explaining interactions between interdisciplinary team members and objects involved in ISD practices. In addition, the typology helps to identify sources of tension between team subgroups.

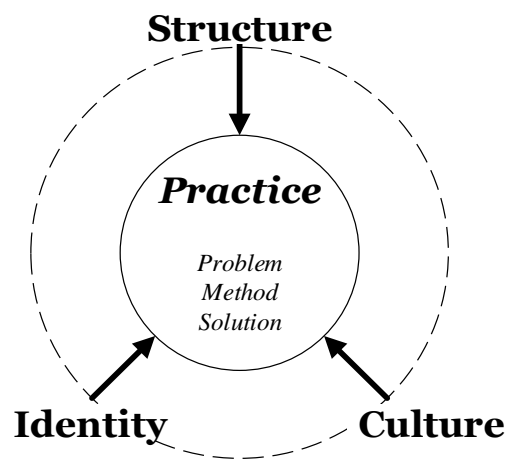

Figure 1. Conceptual Diagram of the Typology for Organizational ISD Practice
The conceptual diagram of the Typology for Organizational ISD Practice is shown in Figure 1. The Typology for Organizational ISD Practice looks at three macro-level constructs which affect social action: Structure, Identity, and Culture. The construct of Structure focuses on positions such as team configurations, roles, and rules. The construct of Identity focuses on the motivations behind an individual's participation in a team and their social action, based on their personal, role-based, and collective interests. The construct of Culture refers to the shared meanings of teams and social groups which influence the value-orientations and assumptions in social processes.

The typology then looks at the ways in which these three macro-level constructs impact team members' shared understanding of and shared commitment to Problem Formulation, Method Formulation, and Solution Formulation in ISD practice. Problem Formulation focuses on deciding the problem to be solved in the field. Method Formulation focuses on deciding the 'modus operandi' of how the team will address the given problem. This may, for example, be a decision to follow an agile approach in ISD. This is influenced by their tacit knowledge, dispositions, and experience. Solution Formulation then focuses on deciding the solution to the aforementioned problem.

Table 1 presents an overview of the Typology for Organizational ISD Practice. A more complete description of the typology can be found in [16]. The typology views structure, identity, culture and human agency (in terms of actions and interactions) as closely interlinked which means that while individuals are subject to macro-level constructs, they still possess agency in how they take action in practice. In addition, the typology views power struggles as being derived from individuals' utilization of economic, social, cultural, symbolic capital [29]. The unit of interest is the concept of practice, which is seen as central to sociomateriality. An embedded unit of analysis focuses on the actions and interactions of team members and objects in this practice. The next section describes the research design of the study.

Table 1. Typology for Organizational ISD Practice

\begin{tabular}{|c|l|l|l|}
\hline Practice & \multicolumn{1}{|c|}{ Structure } & \multicolumn{1}{c|}{ Identity } & \multicolumn{1}{c|}{ Culture } \\
\hline $\begin{array}{c}\text { Problem } \\
\text { Formulation }\end{array}$ & $\begin{array}{l}\text { Examines the influence of } \\
\text { structure on team members' } \\
\text { shared understanding of and } \\
\text { shared commitment to a a } \\
\text { problem formulation. }\end{array}$ & $\begin{array}{l}\text { Exames the influence of } \\
\text { identity on team members' } \\
\text { shared understanding of and } \\
\text { shared commitment to a a } \\
\text { problem formulation. }\end{array}$ & $\begin{array}{l}\text { Examines the influence of } \\
\text { culture on team members' } \\
\text { shared understanding of and } \\
\text { shared commitment to a } \\
\text { problem formulation. }\end{array}$ \\
\hline $\begin{array}{c}\text { Method } \\
\text { Formulation }\end{array}$ & $\begin{array}{l}\text { Examines the influence of } \\
\text { structure on team members' }\end{array}$ & $\begin{array}{l}\text { Examines the influence of } \\
\text { identity on team members' }\end{array}$ & $\begin{array}{l}\text { Examines the influence of } \\
\text { culture on team members' }\end{array}$ \\
\hline
\end{tabular}




\begin{tabular}{|c|l|l|l|}
\hline & $\begin{array}{l}\text { shared understanding of and } \\
\text { shared commitment to a } \\
\text { method formulation. }\end{array}$ & $\begin{array}{l}\text { shared understanding of and } \\
\text { shared commitment to a } \\
\text { method formulation. }\end{array}$ & $\begin{array}{l}\text { shared understanding of and } \\
\text { shared commitment to a } \\
\text { method formulation. }\end{array}$ \\
\hline \multirow{3}{*}{$\begin{array}{c}\text { Solution } \\
\text { Formulation }\end{array}$} & $\begin{array}{l}\text { Examines the influence of } \\
\text { structure on team members' } \\
\text { shared understanding of and } \\
\text { shared commitment to a } \\
\text { solution formulation. }\end{array}$ & $\begin{array}{l}\text { Examines the influence of } \\
\text { identity on team members' } \\
\text { shared understanding of and } \\
\text { shared commitment to a } \\
\text { solution formulation. }\end{array}$ & $\begin{array}{l}\text { Examines the influence of } \\
\text { culture on team members' } \\
\text { shared understanding of and } \\
\text { shared commitment to a } \\
\text { solution formulation. }\end{array}$ \\
\hline
\end{tabular}

\section{Research Design}

An in-depth case study approach [c.f. 33, 34] was chosen to study the information-rich case of an ISD practice. In particular, case studies are well suited to investigating emergent phenomenon such as shared understanding and shared commitment. This in-depth case study focused on a 24-month (May 2015 to May 2017) interdisciplinary connected health project within a distributed environment. The project was a collaborative effort involving partners from industry and academia, including a university research center, a large global technology company, a local SME startup, and a national health insurer. The project was to have two primary outputs; firstly, a new connected health platform to enable the remote monitoring of expectant mothers' wellbeing across different settings such as the expectant mother's home, antenatal clinic, and GP clinic. This platform was to integrate a number of IT artefacts including an Electronic Health Record (EHR), smartphone app, a blood pressure monitor, and urine analyzer. Secondly, a research study was to be conducted involving expectant mothers, using the deployed platform to record symptoms, blood pressure, and urine readings.

A geographically dispersed, interdisciplinary team was formed to produce the first output - the new connected health platform. This team included a Principal Investigator (PI), a clinical lead, a clinical researcher, a research nurse, a project manager, a fulltime and a part-time developer, an analyst, and a data architect. The group was a diverse mix of nationalities, genders, and cultures which can also be seen to impact group decision-making, as per [35].

The lead author was a full-time analyst and active member of the interdisciplinary agile team for a period of six months between June 2015 and January 2016, and he had direct access to the live project environment. Data from the case study was collected and triangulated from three different sources to increase robustness of findings: active engagement (and therefore direct participant observations), interviews, and project documents [c.f. 34]. Participant observations were collected during this time across different locations such as co-design workshops, team meetings, as well as informal conversations. This data was complemented by semi-structured interviews with the ten interdisciplinary team members, with each interview lasting between 60 and 90 minutes. Finally, project documents and emails were used to unearth further insights.

Data was analyzed by the lead author from February 2015 onward using two primary techniques: coding and vignettes. Coding as per $[36,37]$ was used to analyze transcribed interview notes and to organize findings into common themes based on the constructs of the Typology for Organizational ISD Practice. For instance, the lead author adopted a directed approach to content analysis in which the theoretical framework guided the initial codes of interest. The lead author reread the transcript and highlighted text which was representative of predetermined codes. This analysis formed the basis of discussions on the research question. Vignettes as per [37] were used by the researcher to produce, reflect on, and learn from participant observation data and key moments in the 'everyday life' of the project. The vignettes were subdivided according to 'mini-cases' within the case study based on temporal and spatial dimensions as per [c.f. 33, 37]. The next section describes a key vignette from the case.

\section{Vignette of a Design Meeting}

This section outlines a vignette from the case study of the connected health project. The case involves two subgroups [c.f. 38]: 'clinicians' including a clinical researcher and clinical lead, and 'technologists' consisting of a PI, project manager, developer, and analyst. Members of the 'clinician' and 'technologist' subgroups were geographically dispersed across different locations. The vignette of a design specification meeting was selected from the case as it provides a rich account of how the macro-level factors of structure, identity, and culture impacted the microlevel interactions of the project team. It offers a fertile context for investigating the defined research question. Prior to the meeting, the project team had interacted continuously using email, teleconferencing, and a knowledge management system. For instance, the team engaged in online interactions to collaboratively define the project scope, explore different approaches, and 
transfer disciplinary knowledge. Scheduled face-toface meetings were also organized, including a series of workshops to formulate the project vision, and elicit requirements for the platform. However, communication had gradually decreased over recent months of the project, and in the weeks prior to this design specification meeting, interactions between the two groups had all but ceased.

The design specification meeting was scheduled during the fourth month of the project by the project manager in order to reconnect with the clinicians and provide an update on work carried out around the development of the EHR prototype. The meeting took place on October $14^{\text {th }} 2015$ between the hours of 16.00 and 17.45. The project manager, the clinical researcher, the analyst, and a developer attended the meeting. The EHR prototype was an open source solution which had been customized for the purposes of the research study. Certain features of the EHR had been removed and others had been modified or added based on the requirements specified by clinicians during previous meetings and workshops. For instance, the developer had built a Maternity Vitals Assessment form to be used by the clinicians for recording the vital signs of participants in the research study. With deadlines looming, the project manager was keen to get sign-off from clinicians, and to finalize the design specification in line with the project plan.

The vignette is outlined below as a narrative between the team members in attendance. While the PI and clinical lead were unable to attend due to other commitments, their views still shaped the interactions among those present. The narrative has been reconstructed using the lead author's participant observation notes and project documentation.

To begin the meeting, the analyst demoed the changes that had been implemented in the EHR since the team had last met. The team sat around the analyst's computer to discuss the changes.

> Analyst: 'Our work on the 'Maternity Vitals Assessment' prototype form was completed based on the use case requirements. I'll just bring up the form now'. [Analyst moves mouse on PC screen and clicks on option]

$>$ Developer: 'Ok so here on the Maternity Vitals Assessment form, the mandatory fields are the 'Date' and 'Category' field. The 'Category' field is used for categorizing why the assessment has been undertaken and it has four options: 'Routine', 'Post-Op', 'Orthostatic', and 'Unstable'.'

$>$ Clinical researcher: 'The title 'Category' here doesn't make sense for the research study. Could you change the title to 'Location'?'

> Project manager: 'Ok I understand. But I thought the Location would be specified when you're recording details of the participant visit rather than results of the actual assessment? I'd prefer if we could avoid making any unnecessary changes.'

> Clinical researcher: 'The clinical lead would like to see it here. Also, the 'Pulse', 'Cuff size', and 'Position' fields aren't needed. Otherwise its ok.'

These changes were not anticipated by the other team members and contradicted previous discussions on how readings of the vitals are recorded. Once the analyst's demo was concluded and any changes to the requirements were noted, the project manager moved on in the hope that team members could progress towards sign-off.

> Project manager: 'So are we happy with these discussed changes to the EHR? We would hope to close out requirements today as the deadline is approaching.'

> Clinical researcher: 'Yes in general it's fine. The list of Symptoms you showed me are ok, but the clinical lead wants to add 'Birth interval of $>10$ years' and 'Maternity Age > 40' to the Risk Factors list. They would be of interest to the research study'.

> Project manager: 'Ok these factors weren't mentioned before. Do you require any other items to be added to this list?'

> Clinical researcher: 'No I think that's it. The additional risk factors came up during my recent conversations with the clinical lead. She hadn't discussed them with me before either.'

It was becoming apparent that a gap in understanding had opened between the clinicians and the other team members. The clinical researcher did not seem to remember the previously agreed list of symptoms and risk factors, and the analyst had to display both lists to remind her.

$>$ Project manager: 'So is there anything else that we need to change?

> Clinical researcher: 'Is it possible to automatically calculate the gestational age of each participant? I think this is a priority, and should be included before any work is finalized.'

$>$ Project manager: 'We ruled this requirement out of scope at one of the recent workshops.'

$>$ Clinical researcher: 'I think the requirement needs to be ruled back in scope as it will ensure that the gestational age entered is correct. The calculation is currently done manually in the hospital but automating it in the system would help reduce the risk of error. There are smartphone apps that have a gestational age calculator. Can you not take this code and use it?'

> Project manager: 'It's not that straightforward! As I said the requirement was documented as out of scope so ruling it back in at this stage will put a lot 
of pressure on the project development timeline. Also we had previously discussed that values from the paper-based maternity chart should be transcribed verbatim into the EHR.'

$>$ Clinical researcher: 'It's likely that a midwife will be entering data for the research study and if there's an error with the gestational age figure, the clinical lead will ask me why it's inaccurate. This will be avoided if the calculation is automated.'

$>$ Project manager: 'We didn't know that the midwife would be involved. We'll have to extend the deadline to allow enough time to develop this new feature. This impacts on the start date of the research study.'

The clinical researcher's request had come as a surprise to the other team members as their understanding was that the requirement to calculate the gestational age had been ruled out of scope during an earlier workshop. However, the clinical researcher expected that the team would provide flexibility to allow the list of requirements to continue to evolve overtime, and she was also surprised that her request would impact on the timeline. As a result, the atmosphere of the meeting became contentious with both sides failing to reach agreement on how to proceed. At one point the clinical researcher expressed frustration with the discussions.

> Clinical researcher: 'Fine, just get rid of the automated gestational age calculator. I'll calculate it manually.'

$>$ Project manager: 'Hold on, we can explore if it might be possible to reach a compromise. Are there any alternatives to the automated calculation?'

$>$ Clinical researcher: 'Well it would help if there was a field for entering the expected delivery date, but an automated calculator would be better.'

$>$ Project manager: 'But as I said the date for adding new requirements has passed. We want to close out requirements now. If this had been highlighted earlier, we could have built the feature but now we only have a few weeks before the deliverable is due.'

$>$ Clinical researcher: 'But it's important for us that an accurate gestational age figure appears for each participant record.'

Despite the other team members' effort to communicate the difficulty they faced in implementing this requirement within the available time, the clinical researcher asserted that the requirement was essential and a compromise did not seem forthcoming. The clinical researcher then indicated that she was eager to end the meeting and return to obligations in the hospital and she moved towards the door to leave. Before leaving, the team did agree that it would be useful to organize a follow up meeting to run through the EHR's features again. However, a few days after the meeting, the clinical lead emailed the PI and the other team members to say that the requirement to develop a gestational age calculator must be ruled back in-scope. The team was then mandated by the PI to implement the requested feature. Despite their initial disappointment with the decision, the non-clinical team members proceeded to complete the task. The next section discusses insights from the vignette.

\section{Discussion of Findings}

This section discusses findings using the Typology for Organizational ISD Practice as a theoretical lens.

\subsection{The Impact of Structure on Practice}

The problem centered around how to monitor expectant mothers' wellbeing across multiple settings and detect hypertensive disorders of pregnancy. This problem was seen to be relatively tame as prior to the design specification meeting, team members were quickly able to agree the problem formulation and in particular around its clinical and technical aspects. What proved to be interesting was that this activity was done differently across the subgroups. As evident in the vignette, the clinicians adopted a centralized team configuration in which the clinical lead was viewed as an authoritative source for articulating the clinical aspects of the problem. As stated by the clinical lead in an interview: "I articulated what the clinical need was and we worked on putting a proposal and architecture together... the clinical need was well understood." In contrast, the technologists adopted a decentralized team configuration in order to formulate technical aspects of the problem. Due to resource and timeline constraints, the technologists could not afford to wait on a centralized figure to make decisions. As stated by the data architect: "[technologists] wielded extreme power... in the choice of technology and what could be completed within the time scale".

However, friction between the centralized team configuration of clinicians and the decentralized team configuration of technologists contributed to difficulties around finding a method formulation that worked. Technologists felt that the clinicians' formal hierarchy constrained the ability to move forward quickly as the clinical researcher often did not make decisions in the absence of the clinical lead. One developer noted "[the clinician group] is a top down structure and they can't make much decisions without going to the [clinical lead]. The clinical researcher had to ask [the clinical lead] a lot of questions". The clinical researcher also acknowledged this hierarchy: "There's some hierarchy in [the clinician group] and I 
need to consult [the clinical lead] to make decisions. I definitely felt that I was below [the clinical lead]".

The structure of the team also impeded the solution formulation. The clinicians' expertise in obstetrics meant that they were conferred with power over the solution formulation. However, despite this sense of power, clinicians did not see the need to take ownership of project deliverables (e.g. the EHR prototype). For instance, the project manager had hoped that all team members would work together and share ownership of deliverables; however, team members perceived that ownership resided solely with technologists. The project manager observed that "[technologists] were seen to own nearly every deliverable, which was a problem. It might have seemed to give [technologists] power but ... it gave those who didn't [take ownership] even more power to claim the veto on those deliverables". In particular, this lack of commitment was also seen in clinicians' lack of motivation to direct their own time towards some project deliverables.

\subsection{The Impact of Identity on Practice}

The technologists had made significant efforts early in the project to attempt to align team members' identity related interests around the problem formulation. For instance, workshops were organized to facilitate collaborative discussions around the clinical and technical aspects of the problem to be addressed. The project manager indicated that vision building was crucial to aligning team members' interests around the problem: "I think it transpired at some of those early meetings that there wasn't a shared understanding of what [the problem] was all about... So I think at the start it was about taking baby steps... trying to communicate a vision, trying to share that vision and then ultimately trying to align activities around it". Team members also indicated that this vision was a key moment where the problem became structured, well-defined, and the team reached a shared understanding of the problem.

However, shared commitment to a method did not necessarily follow. Technologists felt that the conflict which arose during the design specification meeting had emerged in part due to clinicians' lower level of prior engagement with the method formulation in the proceeding months. For example, during the design specification meeting, the clinical researcher appeared to have forgotten the list of previously agreed data points and requested new requirements which contradicted prior discussions. One developer noted: "[clinicians] don't realize the importance of involvement. They're prioritizing medical work [in the hospital]. We need to engage and collaborate as everyone wants the same goal". Clinicians felt hospital work was always more important than project work however. As stated by the clinical researcher: "if [consideration of] the patient is taken out, there is less of a rush to have to do work, as you are no longer focused on this person and their condition".

Similarly, divergent interests among the clinicians and technologists contributed to difficulties around the solution formulation. In particular, tension between technologists' prioritization of project concerns, and clinicians' focus on hospital concerns constrained the team's ability to formulate an overall solution. For instance, these differences in interests became evident during the design specification meeting when the clinical researcher quickly left to return to matters in the hospital even though there were items to be resolved. One developer noted differences in interests: "Clinicians don't view the project as a development project. They see the project as a research study and plan around what they need to get out of it." Similarly, the clinical researcher stated that "Clinical work is different to project work. I feel [technologists] always think in terms of projects. It is a [change for me] to think in terms of projects".

\subsection{The Impact of Culture on Practice}

In the months prior to the design specification meeting, the technologists had designed various artefacts to help explore cultural differences between team members' shared meanings, values, and assumptions around the problem formulation. These artefacts had included journey maps [c.f. 39], fictional personas of potential participants in the research study, and prototypes of elements of the connected health platform. The technologists felt that their efforts in this respect had been very fruitful and helped increase the team's understanding of the problem early on. As mentioned by the project manager: "there's no doubt that personalizing the journey, using the likes of personas, scenarios, the journey maps were incredibly effective... For me that wasn't by accident... we sat down before each of those workshops trying to figure out how would we break down the barriers".

However, as evident in the design specification meeting, cultural differences constrained the method formulation which in turn compromised shared commitment. For instance, technologists valued a formalized method where requirements were documented and signed off. However, clinicians did not share this commitment. For instance, one developer stated: "The [clinical researcher] didn't realize she was making decisions. We see these as requirements but she sees it as discussions where [things can] change from one day to next. The process was more 
formal than [the clinicians] were used to". Clinicians instead valued a flexible method which could accommodate continuing change overtime. The research nurse later noted: "From the clinical side you'd be used to things being pushed back and not happening on schedule because people might be sick and ... there would be things getting in the way".

Culture differences also created issues around the solution formulation. For instance, clinicians' attitude towards the EHR prototype was questioned by technologists. System logs showed that no attempts had been made by the clinicians to login and review the prototype EHR. As stated by one developer: "The virtual machine is running 5 days a week and it's never used. Clinicians have no curiosity to see what's there... the EHR prototype is not priority until we make it priority". Meanwhile, the clinical researcher felt technologists did not understand the value of the gestational age calculator for clinicians, and as a result were less willing to implement the change: "Certain things are important to clinicians which techies may not understand such as [the] gestational age [calculator]... [technologists] had a different perception of the requirement."

Table 2 summarizes the impact of wickedness on shared understanding and shared commitment.

\section{Table 2. Typology for Organizational ISD Practice Discussion}

\begin{tabular}{|c|c|c|c|}
\hline Practice & Structure & Identity & Culture \\
\hline $\begin{array}{c}\text { Problem } \\
\text { Formulation }\end{array}$ & $\begin{array}{l}\text { Clinicians' centralized team } \\
\text { structure and technologists' } \\
\text { decentralized team structure } \\
\text { did not create wickedness in } \\
\text { the problem formulation and } \\
\text { did not impact shared } \\
\text { understanding and shared } \\
\text { commitment. }\end{array}$ & $\begin{array}{l}\text { Vision building workshops } \\
\text { aligned the interests of } \\
\text { clinicians and technologists, } \\
\text { and created low wickedness in } \\
\text { the problem formulation and } \\
\text { did not impact shared } \\
\text { understanding and shared } \\
\text { commitment. }\end{array}$ & $\begin{array}{l}\text { Designed artefacts such as } \\
\text { journey maps and prototypes } \\
\text { aligned cultural values and } \\
\text { created low wickedness in the } \\
\text { problem formulation and } \\
\text { positively impacted shared } \\
\text { understanding and shared } \\
\text { commitment. }\end{array}$ \\
\hline $\begin{array}{c}\text { Method } \\
\text { Formulation }\end{array}$ & $\begin{array}{l}\text { Tensions between these } \\
\text { centralized and decentralized } \\
\text { team structures created high } \\
\text { wickedness in the method } \\
\text { formulation which negatively } \\
\text { impacted shared } \\
\text { understanding and shared } \\
\text { commitment. }\end{array}$ & $\begin{array}{l}\text { Conflict caused by clinicians' } \\
\text { focus on hospital work at the } \\
\text { expense of project work } \\
\text { created high wickedness in the } \\
\text { method formulation which } \\
\text { negatively impacted shared } \\
\text { understanding and shared } \\
\text { commitment. }\end{array}$ & $\begin{array}{l}\text { Technologists' value on } \\
\text { planning and clinician's value } \\
\text { on flexibility created high } \\
\text { wickedness in the method } \\
\text { formulation which negatively } \\
\text { impacted shared } \\
\text { understanding and shared } \\
\text { commitment. }\end{array}$ \\
\hline $\begin{array}{c}\text { Solution } \\
\text { Formulation }\end{array}$ & $\begin{array}{l}\text { Clinicians' power to veto, and } \\
\text { lack of ownership over } \\
\text { deliverables created high } \\
\text { wickedness in the solution } \\
\text { formulation which negatively } \\
\text { impacted shared } \\
\text { understanding and shared } \\
\text { commitment. }\end{array}$ & $\begin{array}{l}\text { Clinicians' mandate to re- } \\
\text { include the gestational age } \\
\text { calculator created high } \\
\text { wickedness in the solution } \\
\text { formulation which negatively } \\
\text { impacted } \\
\text { understanding and shared } \\
\text { commitment. }\end{array}$ & $\begin{array}{l}\text { Clinicians' lack of } \\
\text { engagement } \\
\text { prototype EHR created high } \\
\text { wickedness in the solution } \\
\text { formulation which negatively } \\
\text { impacted } \\
\begin{array}{l}\text { understanding and shared } \\
\text { commitment. }\end{array}\end{array}$ \\
\hline
\end{tabular}

While prior studies have examined structure, identity, and culture as isolated properties of group decision-making, this study focuses on investigating the dynamic interplay between these constructs to break open the social aspects of wickedness. Our empirical findings suggest that wickedness emerges from the dynamic interplay of these constructs and the resulting wickedness in turn impedes shared understanding and shared commitment.

In addition, our findings suggest that although retrospectively low levels of wickedness were associated with the problem formulation, wickedness still emerged during method and solution formulation. For instance, at face value, the clinical and technical needs appeared structured, which meant that the team were quickly able to reach a shared understanding and shared commitment during problem formulation. However, engaging team members in the method and ensuring ownership of the solution was subject to high levels of wickedness.

This finding challenges extant literature's focus on 'wicked problems' which overlooks the possibility that wickedness can separately be a feature of method and solution formulation. Extant literature on wicked problems, assumes that complexity, uncertainty, and contention in ISD are inherent to the problem to be addressed, rather than being unique characteristics of the activities required to be conducted in the practice of 
ISD [25, 26, 40]. For example, this does not allow for situations in which the problem is relatively tame but wickedness still characterizes how individuals formulate a method and solution to the 'tame problem'. Yet despite this assumption, the wickedness that practitioners and scholars speak of often centers on issues of ISD practice rather than the problem itself. For instance, Yeh's [25] conceptualization of 'system development as a wicked problem' centers almost entirely on wickedness in the ISD practice rather than the problem to be addressed.

This realization prompted the authors to differentiate between wickedness exhibited in the problem (and its formulation), and wickedness exhibited elsewhere in practice. Drawing on the findings of our research, we therefore postulate a complementary approach which identifies nuances of 'wickedness' in practice. For example, we suggest that a practice may be characterized as 'wicked' even where the problem to be addressed is relatively 'tame'. This conceptualization of 'wicked practices' goes beyond Rittel and Webber [12], and suggests that wickedness is not necessarily hinged on the problem (and its formulation) but can equally manifest elsewhere, such as in the method (and its formulation) or the solution (and its formulation). This paper represents what is to the best of our knowledge, the first time that these nuances of wickedness in ISD practice have been drawn out clearly in an empirical and theoretical way. The next section concludes with practical and theoretical implications for ISD practices.

\section{Concluding Remarks}

The emergence of wickedness raises significant barriers to shared understanding and shared commitment in group decision-making processes. For example, wickedness creates fragmentation among social groups which can impede the creation of clear and agreed solutions due to seemingly irreconcilable differences in interpretations. In the absence of shared understanding and commitment, ISD teams risk failure in spite of their best efforts as the implications of wickedness throughout the practice can be hard, if not impossible, to predict. We advocate a deeper understanding of the nuances of wickedness and their impact on managing ISD practice. Our conceptualization of wicked practices is a novel development, one which has important connotations for ISD practice in distributed environments.

From a practical perspective, we are finding that ISD team performance in wicked practices rests on the ability of management to foster argumentative mechanisms and clarify sources of team fragmentation. Wickedness is an emergent phenomenon which cannot be anticipated prima fascia. Therefore, managers should assume an ISD practice is wicked until proven otherwise. This assumption has important practical implications as the management of a wicked practice is inherently different to that of a routine or tame practice. For instance, while structured, linear approaches might be appropriate for a tame practice, it is likely to constrain a wicked practice as formulating a definitive right or wrong approach is not possible. Instead, wicked practices demand an iterative and argumentative response to clarify nuances of wickedness among the team.

From a theoretical perspective, we advocate that further research is needed to investigate the relationship between 'wickedness' and ISD project success in distributed environments. Failure to address social aspects of wickedness in ISD practice can provide another source of explanation for the underperformance of ISD project teams, beyond more technocratic explanations e.g. KPI metrics. However, our research is pointing to a complex relationship between wickedness and ISD project success. For instance, literature from the innovation domain asserts that a divergence of ideas can be advantageous in design teams and help support the development of more innovative and effective artefacts [41, 42]. The management of wickedness in ISD therefore merits further research to understand its relationship with ISD project success.

Acknowledgements: This publication has emanated from research conducted with the financial support of Science Foundation Ireland (SFI) under Grant Number SFI/12/RC/2272.

\section{References}

[1] D. Avison and G. Fitzgerald, ISD: methodologies, techniques and tools, McGraw Hill, 2003.

[2] R. Hirschheim, H. K. Klein and K. Lyytinen, ISD and data modeling: conceptual and philosophical foundations, Cambridge University Press, 1995.

[3] J. F. Nunamaker Jr, M. Chen and T. D. Purdin, "Systems development in information systems research", Journal of management information systems, 7 (1990), pp. 89-106. [4] A. C. Edmondson and I. M. Nembhard, "Product development and learning in project teams: The challenges are the benefits", Journal of product innovation management, 26 (2009), pp. 123-138.

[5] A. Weeger and A. Ott-Schwenk, What Teams Need to Be Clear about-an Activity Theoretical Perspective on Shared Understanding in Health IS Implementation, Wirtschafts Informatik, 2017.

[6] W.-K. Lim, S. K. Sia and A. Yeow, "Managing risks in a failing IT project: a social constructionist view", Journal of the Association for Information Systems, 12 (2011), pp. 374. 
[7] S. Kudaravalli and S. Faraj, Knowledge Collaboration in Distributed Practice Communities, Proceedings from the Thirty Second International Conference on Information Systems, Shanghai, 2011.

[8] M. Hummel, C. Rosenkranz and R. Holten, The Role of Shared Understanding in Distributed Scrum Development:

An Empirical Analysis, European Conference on Information Systems, 2016, pp. 1-18.

[9] P. J. Bateman, P. H. Gray and B. S. Butler, "Research note - the impact of community commitment on participation in online communities", Information Systems Research, 22 (2011), pp. 841-854.

[10] J. Kotlarsky and I. Oshri, "Social ties, knowledge sharing and successful collaboration in globally distributed system development projects", European Journal of Information Systems, 14 (2005), pp. 37-48.

[11] S. Sawyer, P. J. Guinan and J. Cooprider, "Social interactions of information systems development teams: a performance perspective", Information Systems Journal, 20 (2010), pp. 81-107.

[12] H. W. Rittel and M. M. Webber, "Dilemmas in a general theory of planning", Policy sciences, 4 (1973), pp. 155-169.

[13] J. Conklin, Dialogue mapping: Building shared understanding of wicked problems, Wiley, 2005.

[14] R. Buchanan, "Wicked problems in design thinking", Design issues, 8 (1992), pp. 5-21.

[15] B. W. Head, "Wicked problems in public policy", Public Policy, 3 (2008), pp. 101-118.

[16] S. McCarthy, P. O'Raghallaigh, C. Fitzgerald and F. Adam, A Typology for Organizational ICT Practice,

Proceedings of the 50th Hawaii International Conference on System Sciences, 2017.

[17] C.-M. Chiu, M.-H. Hsu and E. T. Wang, "Understanding knowledge sharing in virtual communities: An integration of social capital and social cognitive theories", Decision support systems, 42 (2006), pp. 1872-1888.

[18] R. F. Zammuto, T. L. Griffith, A. Majchrzak, D. J. Dougherty and S. Faraj, "Information technology and the changing fabric of organization", Organization Science, 18 (2007), pp. 749-762.

[19] J. S.-C. Hsu, T.-H. Chu, T.-C. Lin and C.-F. Lo, "Coping knowledge boundaries between information system and business disciplines: An intellectual capital perspective", Information \& Management, 51 (2014), pp. 283-295. [20] J. He, B. S. Butler and W. R. King, "Team cognition: Development and evolution in software project teams", Journal of Management Information Systems, 24 (2007), pp. 261-292.

[21] M. Tan, "Establishing mutual understanding in systems design: An empirical study", Journal of Management Information Systems, 10 (1994), pp. 159-182.

[22] E. A. C. Bittner and J. M. Leimeister, "Creating shared understanding in heterogeneous work groups: Why it matters and how to achieve it", Journal of Management Information Systems, 31 (2014), pp. 111-144.

[23] M. Kleinsmann and R. Valkenburg, "Barriers and enablers for creating shared understanding in co-design projects", Design Studies, 29 (2008), pp. 369-386.

[24] R. O. Briggs, G. L. Kolfschoten and G.-J. d. Vreede,

"Toward a theoretical model of consensus building", AMCIS 2005 Proceedings (2005), pp. 12.
[25] R. T. Yeh, "System development as a wicked problem", International Journal of Software Engineering and Knowledge Engineering, 1 (1991), pp. 117-130.

[26] H. Tanriverdi, A. Rai and N. Venkatraman, "Reframing the dominant quests of information systems strategy research for complex adaptive business systems", Information Systems Research, 21 (2010), pp. 822-834.

[27] P. O'Raghallaigh, D. Sammon and C. Murphy, Theorybuilding using Typologies --A Worked Example of Building a Typology of Knowledge Activities for Innovation,

Proceedings of the 2010 conference on Bridging the Sociotechnical Gap in Decision Support Systems: Challenges for the Next Decade, IOS Press, 2010, pp. 371-382.

[28] T. Parsons, The Social System, Routledge, London, 1951.

[29] P. Bourdieu, Outline of a Theory of Practice, Cambridge university press, 1977.

[30] P. Faulkner and J. Runde, "Technological Objects, Social Positions, and the Transformational Model of Social Activity", Mis Quarterly, 37 (2013), pp. 803-818.

[31] Orlikowski, "Sociomaterial practices: Exploring technology at work", Organization studies, 28 (2007), pp. 1435-1448.

[32] P. O'Raghallaigh, S. McCarthy and F. Adam, Sociomateriality: An Object-Inspired Proposal for IS Scholars, Proceedings of the 25th European Conference on Information Systems, 2017.

[33] K. M. Eisenhardt, "Building theories from case study research", Academy of management review, 14 (1989), pp. 532-550.

[34] R. K. Yin, Case study research: Design and methods, Sage publications, 1994.

[35] S. G. Barsade and D. E. Gibson, "Group affect: Its influence on individual and group outcomes", Current Directions in Psychological Science, 21 (2012), pp. 119-123. [36] A. Strauss and J. Corbin, Basics of qualitative research, Newbury Park, CA: Sage, 1990.

[37] M. B. Miles and A. M. Huberman, Qualitative data analysis: A sourcebook, Sage, Beverly Hills, 1994.

[38] A. Aggarwal, Decision making in diverse swift teams: An exploratory study, 47th Hawaii International Conference on System Sciences (HICSS), 2014, pp. 278-288.

[39] S. McCarthy, P. O'Raghallaigh, S. Woodworth, Y. L. Lim, L. C. Kenny and F. Adam, "An integrated patient journey mapping tool for embedding quality in healthcare service reform", Journal of Decision Systems, 25 (2016), pp. 354-368.

[40] R. O. Mason and I. I. Mitroff, "A program for research on management information systems", Management science, 19 (1973), pp. 475-487.

[41] P. R. Carlile, "Transferring, translating, and transforming: An integrative framework for managing knowledge across boundaries", Organization science, 15 (2004), pp. 555-568.

[42] S. McCarthy, P. O'Raghallaigh, C. Fitzgerald and F. Adam, Impacts of Openness on the Success of Information System Development Research Projects, Proceedings of the 13th International Symposium on Open Collaboration Companion, ACM, 2017, pp. 1-8. 